\title{
External memory: High speed, low speed, and no speed
}

\author{
RUSSELL REVLIN \\ University of California, Santa Barbara, California 93106
}

\begin{abstract}
This paper describes potential constraints on the selection of external memory devices (stimulus sources) and suggests options available to the would-be user of such systems for estimating research requirements and the functional parameters of the available external memory devices.
\end{abstract}

In establishing a microcomputer laboratory, the experimental psychologist is beset with a number of problems concerned with selecting from among a seemingly limitless array of hardware components to meet his/her objectives, which initially may be ill-defined. Papers from this conference have often been helpful in illustrating the functioning of in-place laboratory systems that may be duplicated by others (e.g., Bailey, Ward, Spear, Leatherman, Waite, \& Christian, 1979; Crossman \& Williams, 1978; Santa \& Streit, 1978; these are but a few of the many helpful papers). Some of us have learned first-hand, however, that duplication of other people's computer laboratories may neither satisfy our current objectives nor permit even the satisfaction of a modest set of foreseeable objectives. One reason for this is that similarity in general paradigm does not assure similarity of detail (e.g., timing requirements of the stimulus display). A necessary preliminary to the selection of a computer configuration is a microanalysis and explicit specification of the researcher's specific paradigmatic requirements. One area, in this regard, is the amount of information to be displayed to a subject (e.g., the number of characters in the stimulus displayespecially in reaction time paradigms). A second area concerns requirements for the speed of stimulus presentation (the interstimulus intervals, ISIs, and the intertrial intervals, ITIs). For purposes of establishing microprocessor laboratories, these considerations are not independent. For example, selection of the "stimulus source" device (external memory) requires an analysis of both the timing and storage requirements of the paradigm.

The purpose of the present paper is to describe some potential constraints on the selection of external memory devices and to suggest options available to the would-be user of such systems.

The scope of the discussion is limited to the most popular microprocessor systems: those with 8080 or Z-80 CPUs that are usually associated with S-100 bus systems. The instrumentation is limited to over-the-

I wish to express my appreciation to Richard Parker, who provided desperately needed consultation during the preparation of this manuscript. This work was supported in part by NSF Grant BNS 78-24763. counter, nonexotic, easily interfaced devices about which there is considerable expertise. The treatment is concerned primarily with throughput speed from an external source to a stimulus display and its relation to task demands and computer storage. As a result, some guidelines will be established for the selection of a stimulus and data storage device that readily interfaces with the typical microcomputer.

\section{THE PROBLEM}

The external memory (or stimulus source) can severely constrain the range of feasible studies by limiting the speed with which stimuli can be presented in rapid succession (i.e., the ISI). This can be illustrated by considering a not uncommon computer configuration: an Altair computer with $12 \mathrm{~K}$ of RAM memory (a variant of that described by Santa \& Streit, 1978). Most of the memory is consumed by holding an advanced BASIC language, the operating system, and the program that controls the experiments. Given this configuration, let us assume that the researcher is faced with the task of initiating a sentence-picture verification task, in which the student studies a quantified sentence (e.g., "All of the square figures are filled") and then presses a button after reading the sentence and is ready to examine a collection of squares and triangles that may be filled or empty. If the researcher wishes to vary the time interval between the student's "ready" response and the onset of the collection of objects (displayed on a CRT), then the ISI might fruitfully range between $250 \mathrm{msec}$ to perhaps several seconds delay. The difficulty is that the stimulus collection might contain 400 characters (see Figure 1), which may exceed the speed of the stimulus source device in its ability to input the display characters in less than $1 \mathrm{sec}$.

The naive view might be that the experimenter need only select a stimulus source device that can read 400 characters in $250 \mathrm{msec}$. This approach is in error: Even a high-speed floppy-disk system has difficulty providing the requisite 400 characters in less than $1 \mathrm{sec}$. While these devices can typically read $15,000-30,000$ characters/ sec, their start-up, scanning (track-to-track), and setting 


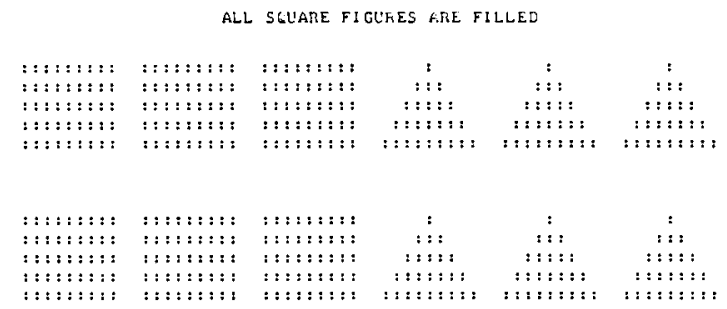

Figure 1. Four-hundred-character CRT display from Revlin and Kallio (Note 1).

times can easily consume $1.0-2.0 \mathrm{sec}$ (these estimates are based on my Micropolis quad-density disk system).

In contrast, some experiments require brief ISIs that can be performed with external memory devices that are quite slow. For example, a typical Sternberg memory scanning experiment (see Aaronson \& Groupsmith, 1978) can be executed with a punch-tape reader attached to an ASR 33 Teletype (Crossman \& Williams, 1978). The ISIs in such experiments tend to be about $2 \mathrm{sec}$, and the number of characters in the test set is typically one. The Teletype punch reads a character every $100 \mathrm{msec}$ (getting-up-to-speed time is difficult to estimate for such devices, but it is on the order of 1 character/ $100 \mathrm{msec}$ ), so that the test item can be transmitted to the CRT in less than $200 \mathrm{msec}$, well within the constraints of the paradigm.

To strain credulity even further, there are circumstances in which a high-speed device, such as a floppy disk, cannot perform optimally and a slow-speed device, such as a Teletype, can function well. For example, in the Sternberg task, after many trials, the test items might be stored in progressively more remote tracks on the disk. Since time to access information increases with the distance that the head must traverse, the retrieval and throughput times might increase so substantially that they exceed the desired ISI.

\section{GENERAL PRINCIPLES}

The foregoing examples are extreme in that they are premised on the absence of additional memory within the microcomputer to store at least a trial's stimuli, to say nothing of the response data. The examples are instructive, however, in illustrating the limitations on the experimental paradigm that are imposed by the external memory device. In cases in which memory residing in the computer is insufficient to store much of the stimuli, the researcher will wish to follow some simple guidelines for selecting an acceptable device for the research program.

The guidelines proceed in three simple steps and are illustrated in Figure 2. First, the researcher will wish to estimate the constraints on the system imposed by the experimental paradigm. This generally has two components: the time available for throughput of the stimuli and the amount of information to be presented. The throughput time parameter $(T)$ is estimated by subtracting the "rise time" of the stimulus device (e.g., a CRT) from the ISI. The rise time is typically the refresh rate for a CRT (maximum time $=16 \mathrm{msec}$, with an average of $8 \mathrm{msec}$ ). The time parameter is then the "time aperture" through which the stimuli can be presented.

The amount of information to be transmitted is also to be calculated, since the rate of information transmission is the basic metric on which external memory devices are evaluated. The researcher will want to calculate the number of characters per ISI to be transmitted to the display device.

The second step is to estimate the limitations on the range of possible external memory devices. This is done by simply subtracting the "access" or "start-up" time for the device from $T$, the time parameter (see above), and multiplying this value by the manufacturer's (not always to be believed) estimate of the "speed" of the device. This provides a functional speed for the external device: functional speed = "speed"(time - access).

The third step, of course, is to compare the paradigmatic constraints with those of the external devices available and select the device that comes closest not only to one's current needs, but, if possible, to one's foreseeable needs, as well.

By way of illustration, consider the sentence-picture verification task described earlier. Here, we have a need to input 400 characters in approximately $.25 \mathrm{sec}$. A floppy-disk system (4-in Micropolis system) can provide approximately 15,000 characters/sec. However, its functional speed is considerably less than this: functional speed $=15,000 \mathrm{cps}(.25 \mathrm{sec}-1.5 \mathrm{sec})$. In this case, the

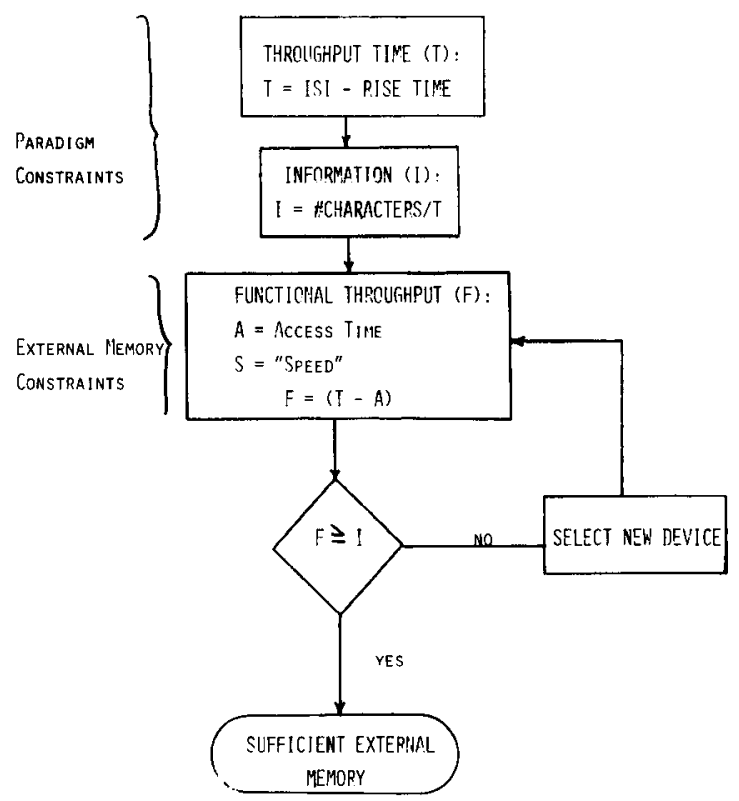

Figure 2. Decision sequence for the selection of external memory devices. 
average time consumed by starting up, scanning tracks, and setting down is approximately $1.5 \mathrm{sec}$. For purposes of the described experiment, the floppy-disk system has a negative functional speed, that is, no speed at all.

However, if the ISI is adjusted so that the 400 characters are needed during an ISI of $4 \mathrm{sec}( \pm 8 \mathrm{msec})$, then the functional speed is equivalent to 37,500 characters during the ISI. In fact, a high-speed reader is also adequate: functional speed $=300 \mathrm{cps}(4 \mathrm{sec}-.1 \mathrm{sec})=1,117$ characters.

These examples are opportune, since they demonstrate the limitations of normally high-speed systems. Clearly, the external memory device is only one component of the entire computer system; the functional speed of such devices varies with the memory milieu of the computer. If ample memory were available in residence (e.g., an extra 16K RAM), the functional speed of the floppy-disk system would be its maximum speed, since most of the stimulus materials could be entered into memory prior to the experimental session. In those cases in which the number of trials is large, planned rest breaks could serve to enter new stimuli during the ITI.

\section{RANGE OF ALTERNATIVES}

The following is a list of potential external memory devices for the typical 8080 system. The devices are listed in order of speed, which also turns out to be close to a chronological order in the manufacture of such devices (from oldest to most recent). The ordering also recapitulates the sequence in which the different systems have been used in my laboratory.

\section{Punch-Tape Reader}

The standard ASR 33 tape reader transmits 10 characters/sec. The rise time (start-up) of the device may be as much as a single character $(100 \mathrm{msec})$. Higher speed Teletypes are available, but see Caveats, below.

\section{High-Speed Tape Readers}

There are a number of these on the market; they tend to be optical scanning and function well at 300 400 characters $/ \mathrm{sec}$. The start-up time of our reader is about $100-200 \mathrm{msec}$. Its speed had to be reduced to assure that characters were not missed by the scanning mechanism.

\section{Cassette Tapes}

These are popular external memory sources and are often provided by the manufacturer as the basic element. The maximum speed of the typical device is between 30 and 150 characters/sec. The functional speed is considerably less than this, since it is typical for records to be stored up to $3 \mathrm{sec}$ apart on the tape to reduce read and write errors.

\section{Floppy Disks}

This has become the external device of choice. When coupled with sufficient memory in residence in the computer, floppy disks provide the opportunity to store both stimuli and responses (usually employing dual-drive systems). Their speeds tend to range from 15,000 characters/sec for 4 -in. disks to 30,000 characters/ sec for the 8-in. disks. The functional speeds vary with both the device and the method of storing the information on the disks. For example, the track-to-track reading times are typically $30 \mathrm{msec}$; if the researcher uses 70 tracks to store the stimuli, it might take several seconds to access the stimuli for a single trial. Functional speed for these devices should take into consideration three parameters: (1) start-up time, (2) track-to-track time, and (3) setting time.

\section{Smart Terminals}

These contain one or more additional pages of memory. Stimuli can be entered into the extra pages while one of the pages is being displayed. The speed of accessing a page is roughly the refresh rate (an average of $8 \mathrm{msec}$ ). Consequently, even a slow external memory can be masked by the effective storage and speed of these terminals. The advantage of smart terminals is limited by the pacing of the presentation of each page in memory. There are instances in which the external device may not be capable of keeping up with the speed of presentation, such as when the display on-time is short due to rapid responding by the subject. The general principles discussed earlier apply to these devices as well as to the standard CRT display terminals.

\section{Resident Memory}

The parameters of the external memory devices can become irrelevant when the internal memory of the computer is optimal, that is, when there is sufficient space in residence to store all stimuli and responses. In general, the access time for RAM memory is faster than the refresh rate of the display devices. With adequate storage, the researcher is free to select external memory devices based on criteria other than speed (see below). In today's market, memory is cheap and represents one of the great bargains in computer-controlled laboratory technology.

\section{CAVEATS}

The focus of this discussion has been on selecting external memory devices to provide optimal throughput speed to meet the needs of the experimental paradigm. There are other criteria upon which external memory devices might be reasonably selected, for example, reliability. Paper tapes jam, wrinkle, and tear. Cassettes drop bits and are subject to the vicissitudes of dirty tape heads and worn drive pulleys. Disk drive heads need to be aligned once per year, and disks wear out. Memory chips burn out and are occasionally difficult to locate. Additional memory boards may unduly stress the power supplies of microcomputers and cause them either to shut down or to intermittently malfunction. All of these systems are fallible. The researcher 
should select them with caution, if not skepticism.

The foregoing guidelines are suggestive rather than exhaustive. They uniformly argue, however, for the importance of detailed specification of research requirements for the laboratory computer and the necessity for careful planning.

\section{REFERENCE NOTE}

1. Revlin, R., \& Kallio, K. Verification of quantified relations. Paper presented at the annual meeting of the Psychonomic Society, St. Louis, Mo., November 1980.

\section{REFERENCES}

Anronson, D., \& Groupsmith, E. The SIMPLE T-scope. Behavior Research Methods \& Instrumentation, 1978, 10, 761763.

Bailey, D., Ward, W., Spear, T., Leatherman, R., Waite, J., \& Christian, T. The CLIPR remote laboratory microprocessor system: Development and applications. Behavior Research Methods \& Instrumentation, 1979, 11, 281-292.

Crossman, E., \& Williams, J. A multi-user on-line 8080 microcomputer system. Behavior Research Methods \& Instrumentation, 1978, 10, 254-258.

Santa, J., \& Streit, P. A microprocessor reaction time laboratory. Behavior Research Methods \& Instrumentation, 1978, 10, 352-355. 